is currently ongoing, and more factors will be studied in the future.

\section{OP49 DEVELOPMENT OF A THEORY-BASED IMPLEMENTATION INTERVENTION TO INCREASE UPTAKE OF DIABETIC RETINOPATHY SCREENING}

${ }^{1}$ F Riordan*, 'E Racine, ${ }^{2} \mathrm{~S}$ Smith, ${ }^{3} \mathrm{~A}$ Murphy, ${ }^{1} \mathrm{~J}$ Browne, ${ }^{1} \mathrm{P}$ Kearney, ${ }^{1} \mathrm{~S}$ McHugh. ${ }^{1}$ School of Public Health, University College Cork, Cork, Ireland; 'Department of General Practice, Royal College of Surgeons in Ireland, Dublin, Ireland; ${ }^{3}$ Department of Economics, Cork University Business School, University College Cork, Cork, Ireland

\subsection{6/jech-2019-SSMabstracts.50}

Background Diabetic retinopathy is a common complication of diabetes affecting the blood vessels at the back of the eye. Despite evidence that diabetic retinopathy screening (DRS) is effective, uptake continues to be sub-optimal in many countries, including Ireland. As routine management of type 2 diabetes largely takes place in primary care, it is arguably the best setting in which to implement interventions to improve DRS uptake. This study aims to develop a theory-based implementation intervention to improve uptake.

Methods A four-stage systematic development process was undertaken. Target behaviours were identified through a multiphase sequential mixed methods study involving key stakeholder interviews $(n=19)$, and an audit of screening attendance in two primary care centres. Barriers and enablers to uptake were identified through coding interviews with patients $(n=48)$ and health care professionals (HCP) $(n=30)$ using the Theoretical Domains Framework (TDF). Barriers and enablers were mapped to behaviour change techniques (BCTs) to develop intervention content. The APEASE (affordability, practicability, effectiveness, acceptability, side effects and equity) criteria was used to select the components. Effectiveness was determined through a rapid evidence review. Feasibility, local relevance and acceptability of the intervention were identified through consensus group meetings with patients $(n=15)$ and HCPs $(n=16)$, and key stakeholder consultation, including the national DRS programme.

Results Three key behaviours were identified; one HCP-level (registration of patients for screening), and two patient-level (consent for the programme to hold their details, and attendance). Modifiable patient barriers and enablers were associated with six TDF domains. Barriers included confusion between screening and routine eye checks ('Knowledge'), forgetting ('Memory, attention, decision processes'), anticipation of a negative result ('Beliefs about consequences'). Enablers included a recommendation from friends/family or HCPs ('Social Influences'), recognising the importance of screening for early detection ('Beliefs about consequences'), ownership over their condition ('Identity'), and being in a routine of attending tests ('Beliefs about capabilities'). HCP barriers included the time to register patients which was impeded or supported by practice resources ('Environmental context and resources'), and a lack information on screening uptake in their local area ('Knowledge'). Following the consensus meetings, consultation and evidence review, ten BCTs were included and operationalised as an implementation intervention targeting professionals (reimbursement, training, audit/ feedback and electronic prompt) and patients (face-to-face/ phone reminder messages, GP-endorsed reminder letter and information leaflet).

Conclusion A multi-stage process combining theory, consultation of multiple stakeholders and existing evidence, was used to develop a multifaceted implementation intervention, targeting both professional and patients, to increase uptake of DRS. The feasibility of delivering the intervention in primary care will be evaluated through a pilot trial.

\section{Ageing/Older People 2}

\section{OP50 COGNITIVE AND SOCIAL ACTIVITIES AND LONG-TERM RISK OF DEMENTIA IN UK WOMEN: PROSPECTIVE STUDY}

S Floud*, A Balkwill, E Mauricio Reus, J Green, GK Reeves, V Beral. Nuffield Department of Population Health, University of Oxford, Oxford, UK

\subsection{6/jech-2019-SSMabstracts.51}

Background Most previous prospective studies have shown an association between lack of participation in cognitive or social activities and increased risk of dementia. However, most studies have been conducted on adults aged over 75 with short follow-up, and thus their findings may be affected by reverse causation bias whereby changes in behaviour are the result of preclinical dementia. In the Million Women Study, we describe the short-term and long-term associations between a lack of participation in cognitive or social activities in women aged 60-65 years old and subsequent risk of dementia.

Methods In 2001, 851,305 UK women, without prior dementia, mean age $60(\mathrm{SD}=5)$ years, reported participation in six social activities (religious group, voluntary work, adult education, art/craft group, music/singing group and bingo); five years later, 645,967 reported hours per day spent doing cognitive activities: reading and watching TV. They were followed by electronic record-linkage to national databases for validated hospital admissions with dementia. Cox regression yielded adjusted relative risks (RRs) for dementia, with follow-up split into 4 year intervals.

Results For the analysis of cognitive activities, 15,529 women had newly-diagnosed dementia over a mean follow-up of 11 $(\mathrm{SD}=2)$ years. During the first 4 years of follow-up, there were apparent excess risks of dementia associated with not reading and not watching TV, but associations weakened substantially with longer follow-up. For women not reading vs reading for $1+$ hours/day, the RRs for dementia reduced from 3.84 (95\% CI 3.19-4.62) in the first 4 years to $1.27(1.14-1.40)$ after $8+$ years follow-up; for not watching TV vs watching TV for $1+$ hours/day, the RRs reduced from $2.18(1.55-3.06)$ in the first 4 years to $1.29(1.09-1.52)$ after $8+$ years follow-up. For the analysis of social activities, 24,437 women had newly-diagnosed dementia over a mean follow-up of 15 years. A lack of participation in any of the six social activities was weakly associated with dementia in the first 12 years of follow-up, with RRs of 1.15 (0.96-1.38), 1.10 (1.01-1.20) and 1.06 (1.01-1.12) during $<4,4-<8$ and $8-<12$ years respectively, but after $12+$ years follow-up there was no association $(0.98,0.94-1.01)$.

Conclusion The associations of dementia with a lack of participation in cognitive and social activities become much weaker 
with longer follow-up and are likely to be largely due to changes in behaviour caused by preclinical dementia.

\section{OP51 PROJECTING THE INCIDENCE AND PREVALENCE OF POST-STROKE COGNITIVE IMPAIRMENT AND DEMENTIA IN THE IRISH POPULATION AGED 40+ YEARS FROM 2015-2025}

${ }^{1}$ E Sexton*, ${ }^{2} \mathrm{NA}$ Donnelly, ${ }^{1} \mathrm{~N}$ Merriman, ${ }^{3} \mathrm{M}$ Guzman-Castillo, ${ }^{3} \mathrm{P}$ Bandosz, ${ }^{2} \mathrm{MA}$ Wren, ${ }^{1} \mathrm{~A}$ Hickey, ${ }^{3} \mathrm{M}$ O'Flaherty, ${ }^{1} \mathrm{~K}$ Bennett. ${ }^{1}$ Division of Population Health, RCSI, Dublin 2, Ireland; ' Social Research, ESRI, Dublin 2, Ireland; ' ${ }^{3}$ Dept of Public Health and Policy, University of Liverpool, Liverpool, UK

10.1136/jech-2019-SSMabstracts.52

Background Post-stroke cognitive impairment (PSCI) is a common consequence of stroke, leading to reduced quality of life and increased care needs. However, rehabilitation services for this condition in Ireland are very limited. The aim was to apply estimates of PSCI incidence to the Irish population and project the number with PSCI in the population in 2025.

Methods We developed a deterministic Markov model to estimate future incidence of PSCI in the population aged 40-89 years living in Ireland up to 2025. Population data, estimates and projections to 2025 were obtained from the Irish Central Statistics Office. Data from the Irish Longitudinal Study on Ageing were used to estimate age and sex specific stroke prevalence in 2014. Age and sex specific stroke incidence was estimated using 2015 public hospital discharge data $(n=6,155)$. Transition probabilities across six health states defined by cognitive impairment, physical disability, dementia and death were estimated using data from stroke survivors in the English Longitudinal Study on Ageing $(n=490)$ (2002-2011). Published data from the South London Stroke Register were used to estimate annual stroke recurrence.

Results The Irish population aged 40-89 years in 2015-2025 $(\mathrm{n}=2.7 \mathrm{~m})$ is projected to have a cumulative incidence of stroke of approximately $2.3 \%$ by $2025(n=63,100)$. Of these incident strokes, approximately $22.5 \%$ are estimated to have died due to stroke $(n=14,200)$, and $23.8 \%$ to have died of another cause $(n=15,000)$ by 2025 . Of the survivors in 2025 $(n=30600)$, approximately $50.9 \%$ are predicted to have cognitive impairment without dementia $(n=15500)$, and $19.4 \%$ to have dementia $(n=5900)$. The total number of stroke survivors is projected to increase from 26700 in 2015 to 41400 in 2025 , equivalent to a $55 \%$ increase in numbers, and the number with post-stroke dementia is projected to more than double from 3900 in 2015 to 8700 in 2025 .

Discussion In 2025, over two thirds of Irish people who have survived a stroke in the preceding 10 years will have cognitive impairment. The number of people with post-stroke dementia is set to double between 2015 and 2025. The model is limited by its deterministic nature, and the assumption that agespecific disease incidence will remain stable. The model will be further developed to include a probabilistic sensitivity analysis, to model alternative scenarios for trends in disease incidence, and to extend the projections to 2035. The model will also be used in an economic evaluation of alternative strategies for stroke management, including cognitive rehabilitation.

\section{OP52 DEMENTIA PROGRESSION IN THE AGEING POPULATION: A COMPUTER SIMULATION ACCOUNTING FOR INDIVIDUAL COGNITIVE AND FUNCTIONAL DECLINE VARIABILITY}

${ }^{1}$ DC Evenden*, ${ }^{1} \mathrm{BM}$ Walsh, ${ }^{2} \mathrm{SC}$ Brailsford, ${ }^{3} \mathrm{PJ}$ Roderick. 'SChool of Health Sciences, University of Southampton, Southampton, UK; ${ }^{2}$ Southampton Business School, University of Southampton, Southampton, UK; ${ }^{3}$ Primary Care and Population Sciences, University of Southamopton, Southampton, UK

10.1136/jech-2019-SSMabstracts.53

Aim To improve planning for the growing older population and the complex care needs of people with dementia, it is important to recognise the variability of individual cognitive and functional decline and associated care costs. However, many studies exploring care demand simplify varied patient trajectories by partitioning dementia patients into severity categories, therefore failing to capture heterogeneity. Our aim was to develop a computer simulation that models dementia progression longitudinally, driven by population-level dementia onset, mortality, and ageing and including individual variability.

Methods Each modelled age group contains two stocks and three flows using the System Dynamics methodology. A cognitively normal (CN) stock is connected via an incident flow to a stock representing people with dementia (PWD). The 65 to 105 year age range is modelled using eight contiguous 5 -year age groups. Ageing is implemented by transferring $\mathrm{CN}$ and PWD survivors from each stock to the next oldest age group at 5 -year intervals. Mortality flows complete the structure for each age group. Published sources provide flow rates. Agentbased methods are used to model individual attributes and outcomes for dementia cases. Progression is modelled by defining a progression rate type for each agent and deriving individual severity progression coefficients. These are based on fixed and random effects regression models, sampled from probability distributions.

Results Mean progression rates are consistent with published studies. By including individualised random effects, the model demonstrates a complex relationship between decline, severity and service use. Rapid decline leads to higher annual care costs with higher mortality rates. Slower decline leads to lower annual care costs over a longer time period. By incorporating these different trajectories within the model, dementia, care costs, and QALYs can be partitioned by fast, intermediate, and slow progression types to more fully support targeted recommendations for planning service delivery.

Discussion Our computer simulation model shows that accounting for the considerable variability in dementia progression rates as well as severity categories provides more accurate representation of the variation in patient trajectories and outcomes. This modelling method hybridises population-level epidemiology and individual-level pathology, allowing future 